\title{
The review of information cognitive Load theory in Online English learning Networks
}

\author{
Yuhong Wang \\ Zhengzhou University of industrial technology, Henan, China
}

\begin{abstract}
The research of information cognitive Load theory in Online English learning Networks refer to many users, it had broad application value and realistic significance to study the problem, The paper introduced the research status about the problem and many works based on four theoretical models, epidemic model, linear threshold model, independent cascade model and game theory model, to research it, and many researchers combined these models to study the problem from impact factors, English learning network structures and competition. At last, The paper summarized and analyzed the worthy of research Problems.
\end{abstract}

Keywords: Online English learning Networks; Information cognitive Load theory; Theory model.

\section{Introduction}

With the popularity of the Internet, online English learning networks (OELN) is widely used in the people's actual life, they can express their views, feelings or communicate with their friends etc.by the way.In essence, the online English learning network is an online community, people can through pictures, text, voice, video and other forms to realize information communication, and remote exchange of views, for example, micro-blogs, blogs, Email has already become an indispensable part of life for many people, it has the function of information cognitive Load theory with characteristics of wide, fast.So it had became an important information source for many people. In China,online English learning networks developed rapidly, to micro-blog as an example, some major web portals have provided micro-blog service, as of 2012, December, the number of Sina micro-blog registered users has reached 500 millions, and has a very important role in promote the cognitive Load theory of related English learning events [1], these events happened in the society often has been discussed extensively and spread in the online English learning networks, and its propagation speed is much faster than the real society. For example, not accurate information about the swine flu was spread rapidly on twitter caused people had great extent panic [2]. In April 6, 2009, a magnitude 3.5 earthquake occurred in Feidong County in China, the message about "the government is urgent purchasing earthquake emergency bag, it will happen a massive earthquake in Hefei"was spread in online socila networks and result serious negative impact on English learning stability [3]. Some enterprises issued on its new products information and some commercial activities and other kinds of information through the form of micro-blog, rapid cognitive Load theory of this kind of information is beneficial to both parties, the vast majority of enterprises were used different forms of English learning networks to spread related business information, including $81 \%$ of enterprises believe that the behavior has a positive effect on the operating results of enterprise [4]. Therefore, different types and different nature of the information abound on the web may result in different degree positive or negative effects to many people, positive and accurate information cognitive Load theory was conducive to English learning stability and economic development, and the effect of negative or error information cognitive Load theory caused to society can not be ignored.

\section{ONLINE English learning NETWORKS}

Online English learning network, referred to as OELN, is a real-time online English learning network, arise after the popularity of the internet application, such as Facebook, twitter, Sina micro-blog etc. Different users purpose with online English learning networks are not same, mainly has the access to information, entertainment, discussion topics, making friends etc. Because of these online English learning networks attract the large number of users, its have hundreds of millions of 
commercial value [5], at the same time, the reason of existence and exponential growth of online English learning networks was the lots of information provided by the users, from the angle of the enterprise to say, a single marketing information achieve to large scale cognitive Load theory must rely on bigger scale and users flow of online English learning network, such as user will share an information from an online English learning network to another English learning network , and different online English learning network users are have different business value to enterprises, such as some influential users and stars, these kind of users may be more effective to affect its fans to forward the already released information, On the other hand, for some ordinary users, they may not be all the time in online English learning networks, their English learning influence is much weaker than star users, so enterprises choose some star users to publish some commodity marketing information is more advantageous clearly, However, compare with the online ocial network scale, the number of a star user followers is still small, if they want to make information cognitive Load theory in large range, it needs more users to participate in the information cognitive Load theory process obviously. In fact, a user may receive a lot of information, the possibility of he can read information is different between other users, even if he can read it, the possibility of forwarding the information is relevant with his preferences, reading behavior, information value judgment and other factors, the user may selective forward similar information that he received, this case may increase difficulty to diffuse information, if there are exist similar or relevant information in online English learning network, these information also exist competition cognitive Load theory in the same online English learning network [6]. Due to the widespread use of online English learning networks, it has attracted many researchers' attention.

Compared to the general English learning network,online English learning networks have some special characteristics. The main content to study OELN $[7,8]$ is that user and network structure of online English learning networks, but the characteristics of network structure has been extensive researched [9], the number of members in traditional English learning network is relatively small,in contrast, user heterogeneity and structure of online English learning network are more complex for example, the average number of close contact with members in the traditional English learning network are between 10 to 20, while the average number of one user's friends in Facebook are 130some experts researched the evolution process of online English learning network,Kumar researched the topological properties of ONS and then proposed a evolution model based on two famous OELN, Flicker and Yahoo360. Wu proposed an online English learning network evolution model based on observation of the growing mechanism in Twitter-like online English learning networks, and then introduced a renewal mechanism for the old nodes and an accelerated growth mechanism for the new nodes, at last, analyzed and numerized topological characteristics of the generated networks and validated properties with some crawled data sets of real online English learning networks. Bu et.al proposed a novel evolution network model with last updating time, which exists in many real-life online English learning networks. The model can maintain the robustness of scale-free networks and improve the network reliance against intentional attacks, and authors also found that it had the small-world effect.Because the user heterogeneity of online English learning networks and information cognitive Load theory involves awide range, there are a large number of sensitive data in online English learning networks may not be conducive to the user, some researchers understand that protect the privacy of users is a very important problem. Most of the OELN is a centralized.

Structure, the service provider has the dominant power and unfavorable to the privacy protection, so some scholars study the distributed structure of OELN (DOELN).

\section{Theory model}

There are rich research achievement about information

Cognitive Load theory, the research value is mainly manifest in viral marketing, control market rumors spread, online advertising and so on. The domestic and foreign literature shows that research mainly carry out theory model, linear threshold model, independent cascade model, the game theory 
model and infectious disease model, to study the subject, and research question focused on maximization information cognitive Load theory and minimization negative information cognitive Load theory, immunization strategy; network structure how to influence information cognitive Load theory and other aspects. Applied theory model to research information cognitive Load theory based on English learning network generally and less use real information cognitive Load theory data as the research object, the main research direction include information cognitive Load theory characteristics, maximization information cognitive Load theory, competitive information cognitive Load theory etc..

\subsection{Epidemic model}

The mechanism of information cognitive Load theory and the spread of infectious diseases is similar, infectious diseases spread through contact by person to person, but information is spread through communication by person to person. A lot of studies about information cognitive Load theory were based on existing research results of the spread of infectious diseases can be thoroughly. The most widely used model is the SIR model and SIS model, first proposed by Kermack and McKendrick respectively in 1927 and 1932. The SIS model divided people into two groups, susceptible state with $\mathrm{S}$ and infected state I, respectively. When susceptible people contact with infected people, susceptible people will be infected with probability $\beta$, and assumed that infected people become too susceptible with probability $\gamma$. The SIR model divided people into three states, susceptible S, infected me and recovery R, similar to the SIS model, when susceptible people contact with infected people, they become infected with probability $\beta$, and infected people become to recovery state with probability $\gamma$. When people become to recovery state and assume that they have permanent immunity and no possibility become to susceptible state again. Figure 1 show the SIS model and SIR model, respectively.
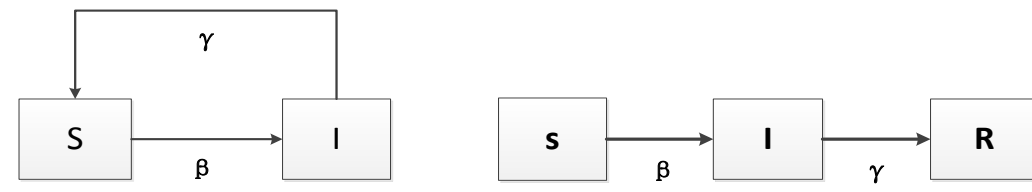

Fig.1. The Model of SIS

\subsection{Linear threshold model}

For each node $\mathrm{v}$ in network, The weight sum of all the edge that connected with node $\mathrm{v}$ need meet the condition $\Sigma_{u \in \nabla} p_{u, v} \leq 1, \nabla$ present the neighbor node set of node v.At the start, part nodes were activated, for any one node, each node was assigned a threshold $\theta_{v}$ between 0 and 1.At time scale t,a non-activated node $\mathrm{v}$ will affected by all activated neighbors of node $\mathrm{v}$, if the sum of influence probability $\mathrm{p}$ of all the neighbor nodes is greater than the threshold of node $\mathrm{v}, \sum_{u \in \mathrm{V}_{\mathrm{t}}} p_{u, v} \geq \theta_{v}$, so the node $\mathrm{v}$ will be activated at time scale $t$, and become activated state at time scale $t+1$, until there is no activated behavior happen and the activated process is terminate.

\subsection{Independent cascade model}

At time scale $t$, each activated node will activate its neighbor nodes at a probability, if a non-activated node is the neighbor of many activated nodes, these nodes will activate non-activated nodes in order, and each activated node activate non-activated nodes only once, whether to activate success, activated nodes no longer to activate these neighbors again, if the neighbor node was success activated, it will activate its neighbor node at time scale $\mathrm{t}+1$.

\subsection{Game theoretical model}

Whether a node accept information decided from the angle of benefit maximization of itself,the node will compare English learning benefit with personal benefit to determine itself maximal benefit.Node make the same choice as its neighbors, it will gain English learning benefit,but personal benefit is that accept information can bring benefit to people.

Few existed research results applied game theoretical to study information cognitive Load theory in online English learning network. 


\section{Conclusion}

When enterprises used online English learning networks for the goal of viral marketing, they often encounter competitors through OELN to promote information cognitive Load theory. From the purpose of enterprise marketing, obviously, each firm hope to make better spread of their information and have a good cognitive Load theory effect. Therefore, there exist information competition cognitive Load theory problems between enterprises, this kind of research achievement is not much also. At the same time, because of the enterprise by the marketing cost constraint and part of marketing information has timeliness, such as corporate promotional information, so research the enterprise marketing information cognitive Load theory problem has a practical significance when consider timeliness and marketing costs.

\section{References}

[1] X. B. Xiong, "Research on the key issues of the communication behavior in micr-blog network", PLA Information Engineering University, 2013.

[2] Ceren Budak, Divyakant Agrawal, Amr El Abbadi, "Limiting the Spread of Misinformation in English learning Networks", Proceedings of the 20th International Conference on World Wide Web, Hyderabad, India, 2011, pp. 665-674.

[3] X. Pan "Research on the communication model of public opinion based on the complex network", Dalian University of Technology, 2010.

[4] M. Stelzner, English learning Media Marketing Industry Report 2009, URL: http://marketingwhitepapers.s3 amazonaws.com/smss09/English learningMediaMarketingIndustryReport.pdf, Accessed: 06/17/2010.

[5] Hoffman, D. L, Novak, T P. "Toward a Deeper Understanding of English learning Media", Journal of Interactive Marketing, vol.26, pp.69-70, February 2012.

[6] Q. Yan, L. Wu, C. Liu, "Information Propagation in Online English learning Network Based on Human Dynamics", Abstract and Applied Analysis, 2013 1-6.

[7] Howard, “Analyzing online English learning networks", Communications of the ACM 51 (11), 2008:14-16.

[8] H. Oinas-Kukkonen, K. Lyytinen, Y. Yoo "English learning networks and information systems: ongoing and future research streams", Journal of the Association for Information Systems, vol.11, pp.61-68, February 2010.

[9] C. Shapiro, H. R. Varian, Information Rules: A Strategic Guide to the Network Economy. Boston: Harvard Business School Press, 1999. 